\title{
Stimulus determinants of brightness and distinctness of subjective contours
}

\author{
SUSAN PETRY, ARDELLE HARBECK, JANE CONWAY, and JUDY LEVEY \\ Adelphi University, Garden City, Long Island, New York
}

\begin{abstract}
Subjective contours are characterized by both the appearance of an illusory border and an increase in apparent brightness within the illusory border. Theories have typically addressed only one of these perceptual attributes. The present experiments measured both apparent brightness and apparent border sharpness of stimuli that varied parametrically in number and width of inducing elements and stimulus configuration. It was found that apparent brightness is influenced more by number of inducing elements, whereas apparent sharpness increases more with inducing element width. Stimulus configuration may influence brightness and sharpness differentially. Thus, to be complete, theoretical accounts of subjective contours must address both perceptual attributes.
\end{abstract}

Normally, a contour is defined as an abrupt change in luminance, wavelength, or purity between adjacent regions in a stimulus array. Under certain favorable conditions, however, contours have been perceived in areas of the visual field where physical stimulation was homogeneous. Schumann (1904) was the first to report the phenomenal appearance of illusory contours in the absence of abrupt stimulus gradients. Subsequently, there emerged a class of phenomena characterized both by the perception of an apparent border and by an apparent increase in brightness defining an illusory figure. The best-known examples of these "subjective contours" are the Kanizsa triangle (Kanizsa, 1955, 1976) and the Ehrenstein illusion (1941). Surprisingly little parametric research has been conducted (Dumais \& Bradley, 1976), although many theoretical interpretations of subjective contours exist in the literature.

One theoretical approach suggests that subjective contours are the result of organizational processes. Some investigators (Kanizsa, 1955; Rubin, 1921; Schumann, 1904) have concluded that subjective contours are the result of organizational processes similar to figure-ground and closure. Others (Bradley \& Dumais, 1975; Gregory, 1972) have proposed cognitive theories in which the observer plays a more active role in the perceptual process. Gregory (1972) states that "illusory contours are the boundaries of objects which are postulated in order to account for blank sectors in the inducing elements" (p. 52). Bradley and Dumais (1975) suggest that they are

We would like to thank David Glosser, Cathy Campenni, and Robin Akerstrom for their help in these studies, and Edward Cochran and Martha Teghtsoonian for their helpful editorial comments. All correspondence should be addressed to: Susan Petry, Department of Psychology, Adelphi University, Garden City, New York 11530 simply "the phenomenal correlates of the edges of visually constructed objects as implied by an analysis by synthesis theory of form perception"' (p. 584). In contrast, Coren (1972), Coren and Theodor (1975), Gregory and Harris (1974), and Lawson, Cowan, Gibbs, and Whitmore (1974) have proposed that subjective contours can be accounted for by the processing of implicit depth cues, resulting in a subjective plane that is stratified in depth relative to the rest of the array, thus producing a simpler overall organization of the percept. The subjective contour, according to this explanation, represents the boundaries or edges of this plane. These apparent boundaries can also function as edges in the production of illusions (Bradley \& Petry, 1977; Meyer \& Garges, 1979). Although these theories account for the perception of an apparent border, they do not directly address the apparent increase in brightness of the subjectively bounded figure.

A second theoretical approach proposes neural explanations of the perceived brightness difference between the subjective figure and its background. Brigner and Gallagher (1974) and Jory and Day (1979) suggest that lateral inhibitory mechanisms can account for the difference in brightness and offer a simultaneous brightness-contrast explanation of the phenomenon. Brigner and Gallagher also emphasize the importance of the interior angle of the inducing elements; Jory and Day also discuss assimilation and end-of-line contrast. A somewhat different lateral inhibitory approach is suggested by Brussell, Stober, and Bodinger (1977). Jung (1973), Jung and Spillman (1970), Spillman, Fuld, and Gerritts (1976), and Stadler and Dieker (1969) proposed theoretical accounts of subjective contours based upon interactions among the receptive fields of orientation-specific cortical cells. These theories explain the apparent increase in brightness defining 
the illusory figure, but fail to account adequately for the perception of an apparent border.

It appears that the theoretical treatment of the phenomenon is limited, addressing one or the other characteristic of a subjective contour but not both. Within this framework, there have been several demonstrations of figures yielding illusory contours without brightness enhancement (e.g., Parks, 1980) and the converse (e.g., Kennedy, 1975, 1976). However, a theory has not yet been formulated to account for figures yielding subjective contours with both an apparent border and an apparent increase in the brightness defining the illusory figure. It is, therefore, important to understand the relation between these aspects of the percept and the stimulus conditions influencing each. In the present set of experiments, we parametrically investigate three spatial variables influencing subjective contour strength-number of inducing elements, size of inducing elements (i.e., percent of subjective contour border defined by the inducing elements), and stimulus configuration. The relationship between these stimulus variables and subjective contour strength can be specified more precisely by viewing them as two experiments. In Experiment 1 , we parametrically explore the effects of the first two stimulus dimensions suggested by Jung and Spillman (1970). The third stimulus variable, stimulus configuration (see Kennedy 1976, 1978a, $1978 b$, and Kennedy \& Ware, 1978), is systematically investigated in Experiment 2.

Thus, the present experiments were conducted for two purposes: (1) to provide a parametric investigation of the spatial characteristics of stimuli generating subjective contours, and (2) to measure, under the same experimental conditions, both the apparent brightness and the apparent distinctness of subjective contours.

\section{EXPERIMENT 1}

\section{Method}

Subjects. Four trained female psychophysical observers with a normal or corrected Snellen acuity of $20 / 20$ participated in this experiment. Three subjects were aware of the purpose of the experiment (J.C., A.H., J.L.); one (T.K.) was naive. The observers ranged in age from 24 to 43 years.

Apparatus. One three-channel Scientific Prototype Model N-100 tachistoscope was used to present the stimuli. The subjects viewed the stimuli, which were located $108 \mathrm{~cm}$ from the eye, binocularly. Stimulus intensity was held constant with a back-ground intensity of $4.35 \mathrm{fc}$ measured by a UDT $80 \mathrm{X}$ photometer.

Stimuli. Seventeen stimuli were used. The inducing elements were drawn in black ink on white $5 \times 7$ in. cards so that the illusory figure appeared in the middle of the field. Figure 1 shows the stimuli used in Experiment 1. All stimuli generated circular subjective contours subtending a visual angle of $1.5 \mathrm{deg}$, because this size was found by Dumais and Bradley (1976) to result in maximum subjective contour brightness. All inducing elements subtended a visual angle of 2 deg and varied in number $(4,8,16$, 32). The percentage of subjective contour border defined by the inducing elements ranged from 5 to 80 . Two stimuli, in which $100 \%$ of the contour's border was defined by the inducing elements, were included as controls.

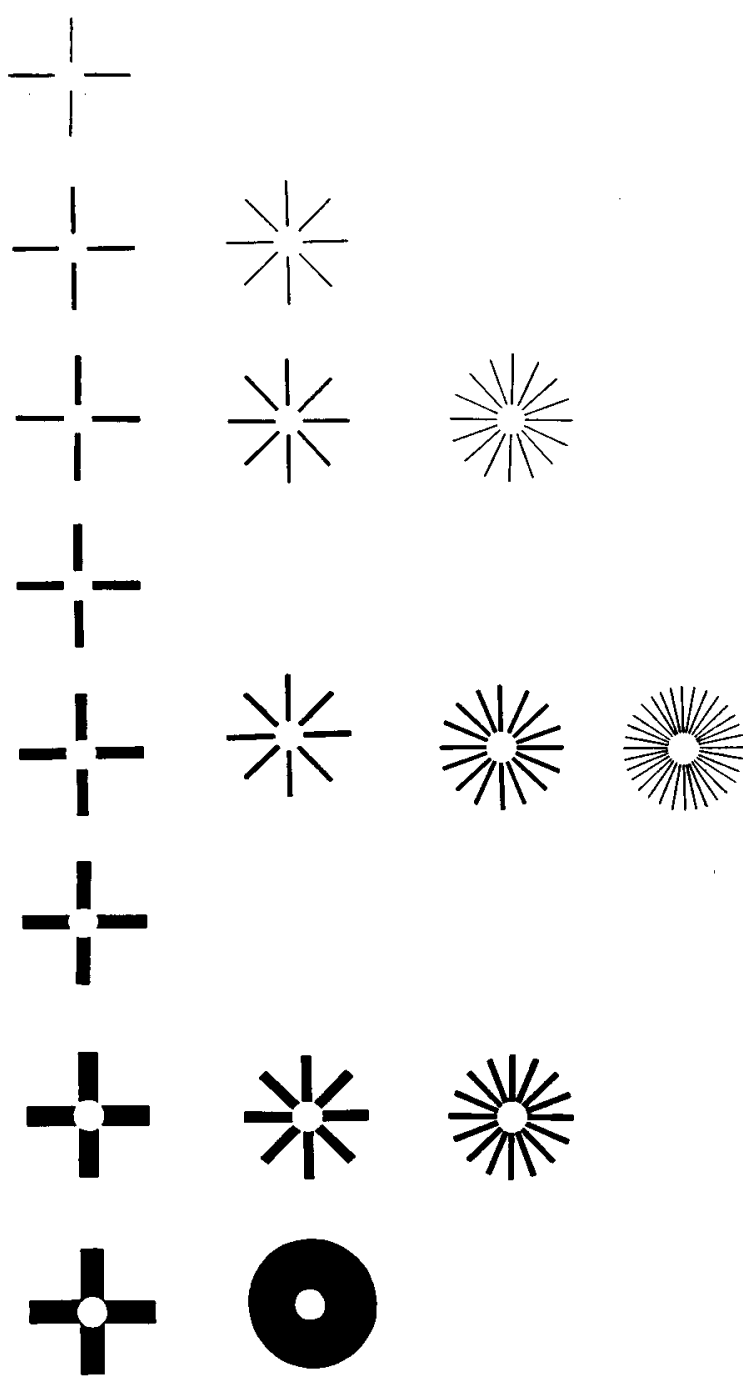

Figure 1. The stimuli used in Experiment 1. The stimuli varied in number of inducing elements $(4,8,16,32)$ and in inducing element width, that is, the percentage of the subjective contour border replaced by the inducing element $(5,10,20,30,40,50$, and 80 , reading down the column of four-element stimuli). In addition, two control stimuli were used: one with four elements replacing $100 \%$ of the contour, and the other an annular stimulus with o.d.-i.d. difference equal to the length of the other inducing elements.

Procedure. Each subject participated in two sessions. Each session was preceded by a 3-min period during which the subject adapted to the background field. The subjects were asked to estimate, in separate sessions, the perceived brightness of the area subsumed by the subjective contour relative to the perceived brightness of the background field, and the contour strength, defined as the distinctness or sharpness of the perceived edge of the illusory contour. A magnitude estimation procedure was used in both sessions: The subjects assigned numbers to the stimuli to match their subjective perceptions of the stimulus dimension under investigation (Stevens, 1957). The method is especially appropriate for use with perceptual variables that are difficult to quantify using objective stimulus manipulations (Petry, 1978; Weisstein, Jerkes, \& Onderisin, 1970). Subjects were initially instructed in the method of magnitude estimation. They used a modulus of " 25 " to indicate brightness of the white background 
field alone, and a modulus of " 50 " to indicate the sharpness of the edge of an inducing element (control stimulus) on the background field. Each stimulus was presented randomly 10 times in each session under a free-viewing condition, and the order of sessions was randomized across subjects.

\section{Results}

The results are shown in Figures 2 and 3. Each data point in Figure 2 represents the mean of the estimates for all four observers. Variability in this experiment and in the subsequent experiment was quite small (perhaps because the subjects were experienced observers), and standard error of the mean was less than the diameter of one symbol. Brightness in all figures is plotted relative to perceived brightness of the background. The brightness ordinate thus represents brightness enhancement, while the perceived sharpness ordinate represents approach to a real contour.

In Figure 2, perceived brightness and sharpness are shown as functions of the percentage of contour defined by the inducing elements, with number of inducing elements as the parameter. Perceived brightness rises to a maximum when about $40 \%$ of contour is defined, then falls slowly as percentage of contour increases; perceived sharpness rises continuously, in a negatively accelerated fashion, as percent contour increases. Perceived brightness shows a maximum variation of $46 \%$ as percent contour changes; perceived sharpness, $700 \%$. Thus, the effect of percentage of defined contour on perceived brightness differs both qualitatively and quantitatively from that which it has on perceived sharpness.

Perceived brightness increases monotonically with number of inducing elements, and the increase is constant across values of percent contour, about $33 \%$. Perceived sharpness seems to increase with number of elements only at lower values of percent contour, at least up to $20 \%$, with no clear effect at values of $50 \%$ or over; the maximum effect of number of con-
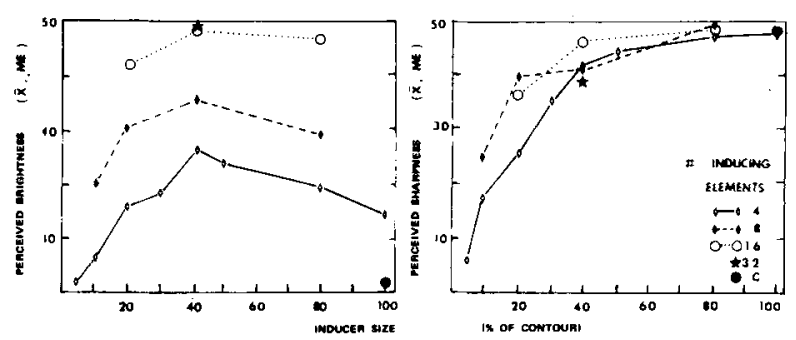

Figure 2. The results of the first experiment. Mean magnitude estimates of apparent brightness (left) and apparent sharpness (right) for all four observers is plotted as a function of inducing element width with number of inducing elements $(0,4 ; 0,8 ; 0$, 16; and $\star, 32$ ) as the parameter. Apparent brightness is plotted relative to background field brightness, which was judged by the observers to be " 25 ." Each data point represents the mean of 40 observations. Standard error of the mean was never greater than the diameter of one symbol.

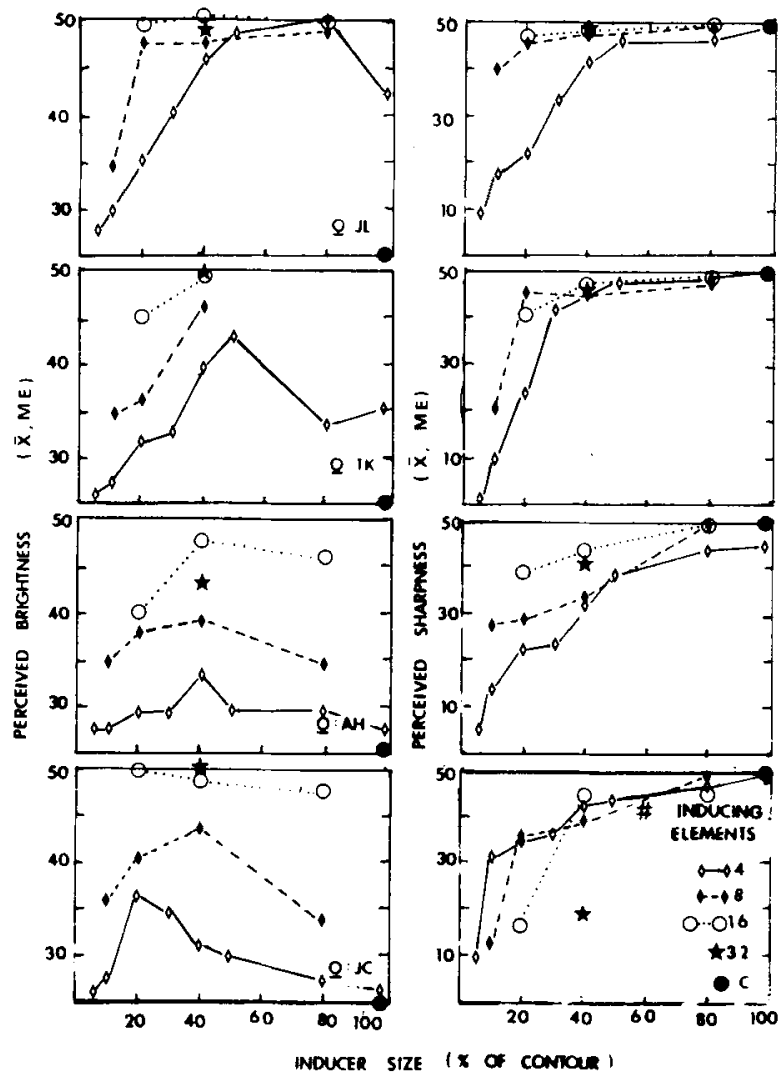

Figure 3. The results of the first experiment plotted for each individual observer. Axes and symbols are the same as in Figure 2. Each data point represents the mean of 10 observations, and standard error of the mean is less than the diameter of one symbol.

tours is an increase in perceived sharpness of $50 \%$ when defined contour is at $20 \%$, but that effect falls to zero at higher levels of defined contour. The effect of number of inducing elements also differs, both qualitatively and quantitatively, for perceived brightness and perceived sharpness.

Thus, perceived sharpness and brightness appear to be determined by different aspects of the stimulus. Perceived brightness is influenced more by the number of inducing elements, whereas inducer size is the major determinant of perceived sharpness. This pattern is also clearly evident in the functions obtained from the data of the individual observers presented in Figure 3. Each data point represents the mean of 10 observations. It is interesting to note that the inducing element size that results in maximum perceived brightness varies from $30 \%$ to $60 \%$ between observers. The relative importance of stimulus dimensions varies as well, but the major conclusions can be seen in the data of each individual observer.

\section{EXERIMENT 2}

The results of the first experiment imply a simple relationship between the stimulus dimensions investi- 
gated and subjective contour strength which may be somewhat misleading. The literature, however, alludes to one other stimulus variable that might influence the strength of subjective contours. In a series of articles, Kennedy (1976, 1978a, 1978b), Kennedy and Lee (1976), and Kennedy and Ware (1978) have demonstrated several circular subjective contours induced from stimuli that differ from those investigated in Experiment 1 . Collectively, they can be organized according to two configurational properties, variable contrast and orthogonality. Variable contrast stimuli may be simply defined as stimuli in which the contrast between the inducing elements and the background field is not constant (i.e., increases from center to end). Orthogonality, with respect to subjective contours, may be defined as stimuli in which the inducing elements are not at right angles to the tangent of the edge of the subjective contour. The effects of the configuration dimension upon subjective contour strength have been unexplored. Therefore, in Experiment 2 we investigated this third stimulus dimension.

\section{Method}

The subjects, stimulus-generating equipment and conditions, and procedure were the same as in Experiment 1.

The stimuli were constructed in an identical fashion to those in Experiment 1. The 17 stimuli used are shown in Figure 4. Constant and variable contrast stimuli (stimuli in which the contrast of the inducing elements were and were not constant, respectively) had an inducing element size of $40 \%$, but varied in number $(4,8$, and 16$)$. The density of the innermost portion (0.5-deg region immediately surrounding the subjective contour) of the variable (VD) and constant (CD) density stimuli was identical.

The orthogonal stimuli consisted of two types of configurations, star-like (ST) and triangular (TR) figures. The inducing element angle differed in the two configurations, being more acute for the triangular than for the star-like patterns. In addition, the subjective contour would probably be perceived as part of the figure in the star-like patterns, whereas in the triangular pattern it would be part of the ground. Therefore, a third configuration pattern (FL) was included in which the interior inducing element angle was identical to that of the triangular pattern but the subjective contour would be part of the figure. Appropriate solid (S) inducing element stimuli from the first study were employed for comparison purposes, and the four-element control stimulus was also included.

\section{Results}

The average results of the four observers are shown in Figure 5. Perceived brightness and sharpness are plotted as a function of stimulus configuration, with number of inducing elements as a parameter. In general, stimuli with inducing elements that are not orthogonal to the subjective contour are less effective than those that are. In particular, they are less effective with respect to perceived sharpness. In fact, only the 16-element stimuli generated any noticeable subjective contours.
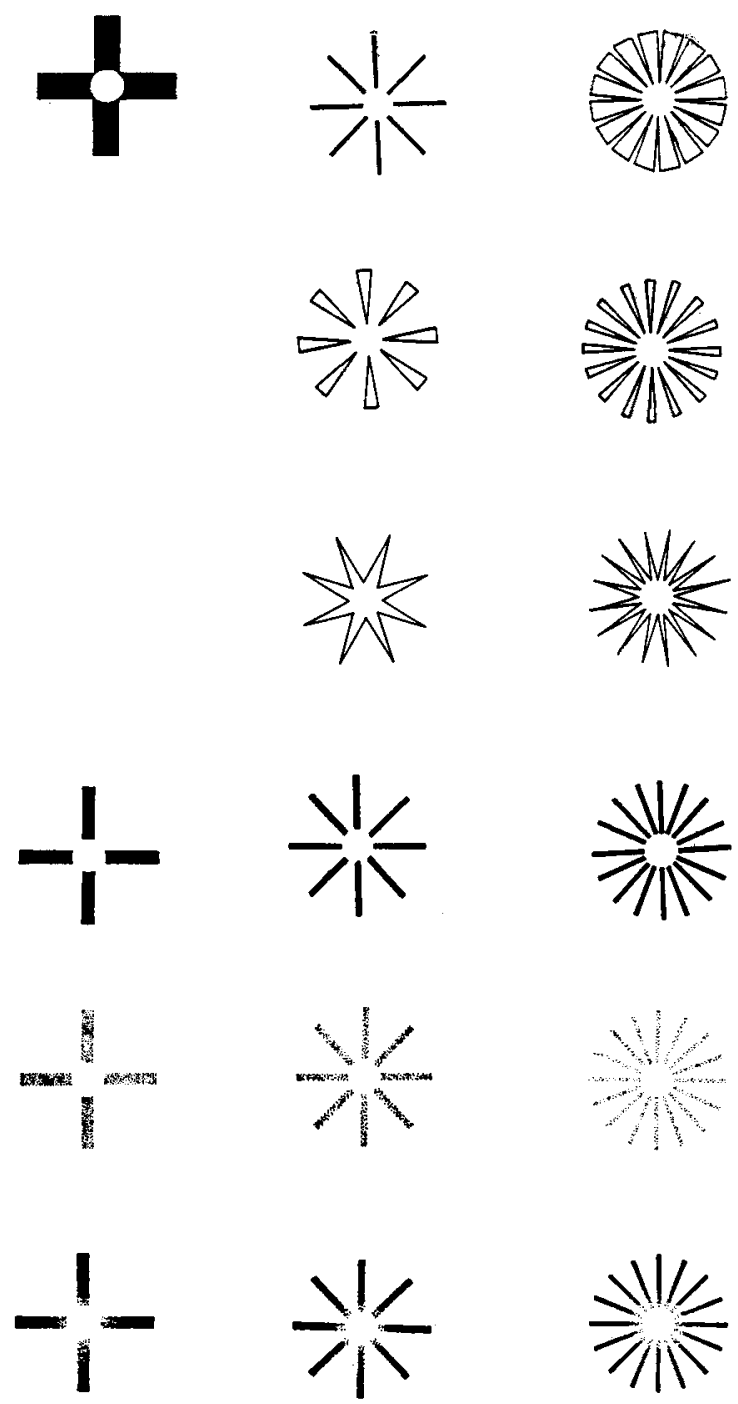

Figure 4. The stimuli used in Experiment 2.

Interestingly, although there is a difference in brightness and sharpness in the "star" and "triangular" patterns, little difference in perceived brightness and sharpness is found between the triangular pattern and the acute-angle star when interior angle is held constant. That is, for these stimuli, local, rather than global, stimulus variables appear to be determining subjective contour strength. These data lend support to Brigner's characterization of subjective contours which emphasizes the importance of the angle formed by the inducing elements.

The most striking result came from the variable contrast stimuli Although the variable and constant density stimuli were identical in the 0.5 -deg region surrounding the subjective contour, perceived sharpness was always greater in the constant density situa- 


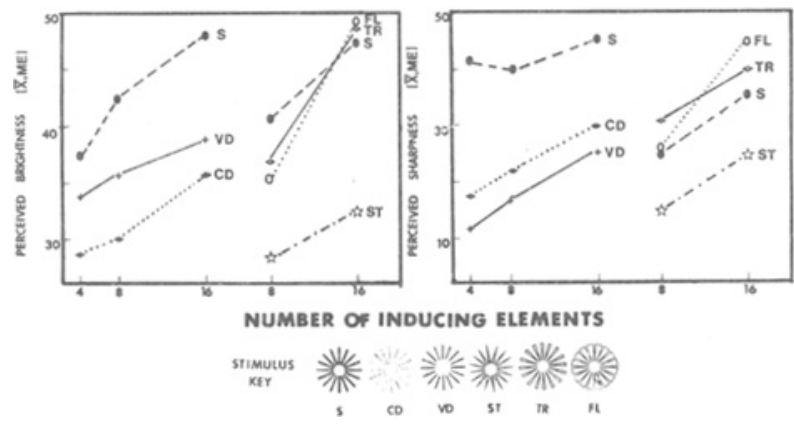

Figure 5. The results of the second experiment. Mean magnitude estimate of apparent brightness (left) and sharpness (right) in arbitrary units for all four observers is plotted as a function of number of inducing elements with stimulus configuration as the parameter. Each data point represents the mean of $\mathbf{4 0}$ observations, and standard error of the mean is less than the diameter of one symbol.

tion. Perceived brightness, however, was greater with the variable density stimuli. Similar patterns are revealed in the functions obtained for the individual observers. Thus, for the variable contrast stimuli, global, rather than local, stimulus variables appear to be determining subjective contour strength.

\section{GENERAL DISCUSSION}

One of the more interesting findings of the studies is that brightness varies as an inverted-U-shaped function of the size of the inducing element. The peak of the function varied between individuals and corresponded to an interinducing element width (gap size) of 10 to $30 \mathrm{~min}$. This implies that brightness perception is mediated by a local receptive-fieldlike mechanism with a limited excitatory area. Such inferred physiological units are known to vary in size across individuals (Matthews, 1971; Petry, 1978). Moreover, the visual angles involved agree fairly closely with psychophysical estimates of human parafoveal receptive field size (Jung, 1973). Thus, local mechanisms are strongly implicated, even though these studies were not designed to determine their nature. The contributions of two potential mechanisms, end-of-line and brightness contrast, may be distinguished from each other, since one would expect more end-of-line contrast when each inducing element was narrow and more brightness contrast when each inducing element was wide. Overall perceived brightness is greater when inducing elements are narrow. Moreover, if gap width is held constant, increasing inducing element width results in decreases in perceived brightness. Thus, perceived brightness of subjective contours is increased more by end-of-line than by brightness contrast mechanisms.
Since increasing the number of inducing elements (decreasing gap size) results in strong increases in perceived brightness, assimilation effects (Helson \& Rohles, 1959; Ware, 1981) may also be present. Such effects probably operate in conjunction with end-ofline contrast. They are not seen when comparing larger diameter inducing elements. (For example, four, i.e., $40 \%$, compared with eight, i.e., $80 \%$, illustrates a situation in which inducing element diameter is relatively wide and constant, and as numbers of inducing elements increases gap width decreases. Perceived brightness, however, remains unchanged.)

The effects of local stimulus variables are seen even more clearly in the results of Experiment 2, in which inducing element angle was varied. Brightness, and to a lesser extent apparent sharpness, were determined in large part by (1) inducing element angle (smaller or more orthogonal interior angles were judged to be brighter), and (2) number of inducing elements. All of these factors are consistent with the hypothesis that end-of-line contrast is an important operating mechanism. Stimulus configuration, that is, whether the subjective contour is perceived on figure (FL, ST) or ground (TR, S), seems to have little effect on subjective contour strength. Parametric research varying interior angle and configuration is needed to answer this question completely.

Global stimulus features or processing mechanisms cannot be completely ignored, however. Subjects frequently reported stratification and figure-ground reversals, especially with prolonged viewing and steady fixation. Apparent sharpness judgments approached asymptote when the inducing elements subsumed $40 \%$ to $50 \%$ of the total figure. Werner (1935) varied percent of mask-test edge congruity in a metacontrast paradigm. He found complete masking when the mask was adjacent to $40 \%$ or more of the test, an effect that has been termed "edge incorporation."

Not surprisingly, reducing contrast (density) of the inducing elements reduces both apparent brightness and sharpness of the subjective contour. Varying contrast (Condition VD in Experiment 2) a way that made the local features (inner third of each inducing element) identical with those of the lower contrast stimuli (Condition $C D$ in Experiment 2) resulted in a subjective contour that was perceived as both brighter and blurrier than its constant lower contrast counterpart. Some subjects, in fact, referred to the variable density figures as "sunbursts." The importance of total stimulus configuration to subjective contour brightness is quite evident with these figures.

In conclusion, apparent sharpness of the subjective contours may be characterized as the result of global processing as posited by Coren and Gregory, while apparent brightness, influenced predominantly by the number of inducing elements, can more easily 
be described by an assimilation, end-of-the-line, or local brightness contrast theory such as those suggested by Kennedy, Jory and Day, Jung and Spillman, and Parks. It should be noted, however, that the variable density stimuli provide an interesting and potentially theoretically important exception to this characterization. Thus, depending on the aspect of the subjective contour in which one is interested, both types of theories may be correct.

In summary, we have shown that perceived brightness and sharpness are two semi-independent components of subjective contours. They seem in large measure to be determined by separate aspects of the stimulus. Perceived brightness depends both on the number of inducing elements and the inducing size, increasing monotonically with the former, and increasing then decreasing with the latter. Perceived sharpness is largely controlled by the inducer size, although number of inducing elements has a small effect. These principles do not appear to place any constraints on the current theories of subjective contours, but they do suggest that a complete theory of subjective contours should address factors contributing to both perceived brightness and perceived sharpness.

\section{REFERENCES}

Bradeey, D. R., \& Dumais, S. T. Ambiguous cognitive contours. Nature, 1975, 257, 582-584.

Bradley, D. R., \& Petry, H. M. Organizational determinants of subjective contour. American Journal of Psychology, 1977, 90, 253-262.

Brigner, W. L., \& Gallagher, M. B. Subjective contoür: Apparent depth or simultaneous contrast? Perceptual and Motor Skills, 1974, 38, 517-524.

Brussell, E. M., Stober, S. R., \& Bodinger, D. M. Sensory information and subjective contour. American Journal of Psy. chology, 1977, 90, 145-156.

Coren, S. Subjective contour and apparent depth. Psychological Review, 1972, 79, 359.

Coren, S., \& Theodor, L. H. Subjective contour: The inadequacy of brightness contrast as an explanation. Bulletin of the Psychonomic Society, 1975, 6, 87-89.

Dumais, S. T., \& Bradley, D. R. The effects of illumination level and retinal size on the apparent strength of subjective contours. Perception \& Psychophysics, 1976, 19, 339-345.

Ehrenstein, W. Über Abwandlungen der L. Hermannschen Helligkeit-Serscheinung. Zeitschrifte für Psychologie, 1941, 150, 83-91.

Gregory, R. L. Cognitive contours. Nature, 1972, 238, 51-52.

Gregory, R. L., \& HarRIs, J. B. Illusory contours and stereo depth. Perception \& Psychophysics, 1974, 15, 411-416.

Helson, H., \& Rohles, R. H. A quantitative study of the reversal of classical lightness contrast. American Journal of Psychology, 1959, 72, 530-538.

JoRY, M. K., \& DAY, R. H. The relationship between brightness contrast and illusory contours. Perception, 1979, 8, 3-9.
Jung, R. Visual perception and neurophysiology. In R. Jung (Ed.), Handbook of sensory physiology (Vol. 1(3)): Central processing of visual information. Berlin: Springer-Verlag, 1973.

Jung, R., \& SpIllman, L. Receptive field estimation and perceptual integration in human vision. In F. A. Young and D. B. Lindsey (Eds.), Early experience and visual information processing in perceptual and reading disorders. Washington, D.C: National Academy of Sciences, 1970.

KanizsA, G. Margini quasi-percettivi in campi con stimolazione omogenea. Revista di Psicologia, 1955, 49, 7-30.

Kanizsa, G. Subjective contours. Scientific American, 1976, 234,(4), 48-64.

Kennedy, J. M. Depth at an edge, coplanarity, slant depth, change in direction and change in brightness in the production of subjective contours. Italian Journal of Psychology, 1975, 2, 107-123.

KEnNEDy, J. M. Sun figure: An illusory diffuse contour resulting from an arrangement of dots. Perception, 1976, 5, 479-481.

Kennedy, J. M. Illusory contours not due to completion. Perception, 1978, 7, 187-189. (a)

Kennedy, J. M. Illusory contours and the ends of lines. Perception, 1978, 7, 605-607. (b)

KENNEDY, J. M., \& LEE, H. A figure-density hypothesis and illusory contour brightness. Perception, 1976, 5, 387-392.

KenNedY, J. M., \& W ARE, C. Illusory contours can arise in dot figures. Perception, 1978, 7, 191-194.

Lawson, R. B., Cowan, E., Gibbs, T. D., \& Whitmore, C. G. Stereoscopic enhancement and erasure of subjective contours. Journal of Experimental Psychology, 1974, 103, 1142-1146.

MatThews, M. L. Spatial and temporal factors in masking by edges and disks. Perception \& Psychophysics, 1971, 9, 15-26.

Meyer, G. E., \& Garges, C. Subjective contours and the Poggendorff illusion. Perception \& Psychophysics, 1979, 26, 302-304.

Parks, T. E. Subjective figures: Some unusual concomitant brightness effects. Perception, 1980, 9, 239-241.

Petry, S. Perceptual changes during metacontrast. Vision Research, 1978, 18, 1337-1341.

RuBin, E. Visuell wahrgenommene Figuren. Copenhagen: Gyldendal, 1921.

SCHUMANN, F. Einige Beobachtungen über die Zusammenfassung von Gesichtseindrucken zu Einheiten. Psychologische Studien, 1904, 1, 1-32.

Spillman, L., Fuld, K., \& GerritTs, H.J.M. Brightness contrast in the Ehrenstein illusion. Vision Research, 1976, 16, 713-719.

Stadler, M., \& Dieker, J. Untersuchungen zum Problem virtueller Konturen in der visuellen Wahrnehmung. Zeitschrift für Experimentelle und Angewandte Psychologie, 1969, 19, 325-350.

Stevens, S. S. On the psychophysical law. Psychological Review, 1957, 64, 153-181.

WARE, C. Subjective contours independent of subjective brightness. Perception \& Psychophysics, 1981, 29, 500-504.

Weisstein, N., Jerkes, T., \& ONDERISIN, T. Effect of forcedchoice versus magnitude estimation measures on the waveform of metacontrast functions. Journal of the Optical Society of America, 1970, 60, 978-980.

Werner, H. Studies on contour: 1. Qualitative analyses. American Journal of Psychology, 1935, 47, 40-64.

(Manuscript received July 26, 1982; revision accepted for publication March 29, 1983.) 